\title{
La periferia: fragmentos inestables de la ciudad vivida
}

THE PERIPHERY: UNSTABLE FRAGMENTS OF THE CITY LIVED

A PERIFERIA: FRAGMENTOS INSTÁVEIS DA CIDADE VIVIDA

\section{Alicia Lindón ${ }^{1}$}

Para citar este artículo: Lindon, A. (2020). La periferia: fragmentos inestables de la ciudad vivida. Perspectiva Geográfica, 25(2) 15-33. https://doi.org/10.19053/01233769.10548
Recepción:

28 de enero de 2020

Evaluación:

28 de abril de 2020

Aprobación:

18 de junio de 2020

\section{Resumen}

En este texto se presenta una aproximación teórico-metodológica a la periferia desde las experiencias espaciales cotidianas de sus habitantes. Ello supone una inversión múltiple del punto de vista frecuente en los estudios urbanos. Por un lado, el espacio y el tiempo se conciben en términos vividos.

Por otro lado, se recurre a una inversión metodológica al tomar como aproximación a la realidad geográfica las narrativas de vida de los habitantes, es decir, los discursos sobre los lugares en vez de priorizar el registro de los lugares en sí mismos.

1 Profesora e investigadora titular del Departamento de Sociología de la Universidad Autónoma Metropolitana, unidad Iztapalapa (Ciudad de México), en el área de investigación de sociología de la cultura y en el cuerpo académico Espacio Social de la Ciudad. Es miembro del Sistema Nacional de Investigadores del Consejo Nacional de Ciencia y Tecnología en el nivel III. Doctora en Sociología por el Centro de Estudios Sociológicos de El Colegio de México. Correo: alicia.lindon@gmail.com. Orcid: https://orcid.org/0000-0002-8424-4322. 
Para ello, en la primera parte se aborda la periferia como fragmentos densos que expresan estados inestables de lo urbano, siempre marcados por la tensión entre el cambio y el orden periféricos. Luego, la propuesta explora el abordaje de la periferia vivida a partir del análisis de las experiencias espaciales de los habitantes y sus biografías. Ello se revisa a través del anclaje periférico de los eventos biográficos de los habitantes de la periferia, tanto individuales como familiares, y también de la configuración de cronotopos periféricos en las narrativas locales.

Palabras clave: experiencias espaciales, habitar, autobiografías, cronotopos.

\section{Abstract}

This paper presents a theoretical-methodological approach to the periphery from the daily spatial experiences of its inhabitants. It implies a multiple inversion of the common point of view of urban studies. On the one hand, space and time are conceived as lived. On the other, the method is inverted by taking the inhabitants' life narratives to approach the geographical reality, i.e., the discourses on places rather than the records of places. The first part addresses the periphery as dense fragments that express unstable states of urban areas, always marked by the tension between peripheral change and order. Then, the proposal explores the approach to the periphery lived from the analysis of the inhabitants' spatial experiences through the peripheral anchoring of their individual and family biographical events and the establishment of peripheral chronotopes in local narratives.

Keywords: Spatial experiences, inhabiting, autobiographies, chronotopes.

\section{Resumo}

Neste texto apresenta-se uma abordagem teórico-metodológica à periferia a partir das experiências espaciais cotidianas de seus habitantes. Isso supõe uma múltipla inversão do ponto de vista frequente nos estudos urbanos. Por um lado, o espaço e o tempo concebemse em termos vividos. Por outro lado, utiliza-se um investimento metodológico ao tomar as narrativas da vida dos moradores como uma aproximação com à realidade geográfica, ou seja, os discursos sobre os lugares ao invés de priorizar o registro dos lugares em si mesmos.

Para o efeito, na primeira parte a periferia é abordada como fragmentos densos que expressam estados instáveis do urbano, sempre marcados pela tensão entre a mudança 
e a ordem periférica. Logo, a proposta explora a abordagem da periferia vivida a partir da análise das experiências espaciais dos moradores e de suas biografias. Isso é revisto por meio da ancoragem periférica dos acontecimentos biográficos dos habitantes da periferia, tanto individuais quanto familiares, e também pela configuração de cronotopos periféricos nas narrativas locais.

Palavras-chave: experiências espaciais, habitar, autobiografias, cronotopos.

\section{Introducción}

Los recientes estudios urbanos atienden un espectro muy amplio de problemáticas que no cesan de ampliarse, siempre asociadas a la ciudad y la vida urbana. Los énfasis disciplinarios son diversos y no pocas veces son transdisciplinarios. Por su misma heterogeneidad, no sería posible ni una mínima enumeración de ellos. Dentro de ese extenso horizonte analítico de lo urbano se subrayan dos cuestiones de particular interés para este texto. Una es la continua expansión del tejido urbano en el territorio, con la consecuente movilidad espacial cotidiana exacerbada, y la otra, todo lo relacionado con las conexiones entre los lugares y las personas dentro de dicho territorio. Ambas problemáticas también han sido tratadas desde diversas miradas. La primera de ellas ha sido revisada particularmente como la conformación de las periferias por el mercado de suelo urbano, la vivienda y sus políticas, y, más recientemente, las formas de segregación y fragmentación urbanas, incluso la formación de archipiélagos urbanos que no pocas veces se asocian a estigmatizaciones territoriales, sin olvidar las guetizaciones. Y en el caso de la segunda - conexiones entre los lugares y las personas en el tejido urbano-, han sido frecuentes los análisis desde la movilidad espacial, la simultaneidad, la aceleración y las innovaciones tecnológicas ${ }^{2}$. Sin menospreciar la relevancia de estos abordajes, el texto que aquí se presenta busca otro acercamiento que complemente el conocimiento de ambas problemáticas. Por un lado, la expansión urbana se retoma a través de la focalización en la periferia como espacio vivido, antes que hacerlo en el horizonte del proceso de expansión metropolitana por el que van emergiendo nuevas periferias en territorios cada vez más lejanos a las áreas centrales. Por otra parte, la relación entre las personas y los lugares interesa en términos experienciales (es

2 Por ejemplo, el dossier del n 132, vol. 44 de 2018 de la Revista EURE estuvo dedicado a la vivienda y el desarrollo inmobiliario. El dossier del n 133, vol. 44 de 2018, también de la Revista EURE, estuvo dedicado a la economía en la configuración urbana. La misma revista, en su n 134, vol. 45 de 2019, dedicó el dossier a la accesibilidad y la movilidad en la planificación urbana. Por su parte, la revista Urban Studies identifica como uno de sus artículos recientes más consultados, publicado en 2019, el que lleva por título "Measuring and mapping displacement: The problem of quantification in the battle against gentrification", https:// doi.org/10.1177\%2F0042098019851953. Entre sus artículos recientes, también cabe destacar el que lleva por título "An international housing market in the British Isles: Evidence from business and medium-term cycles using a Friedman test", publicado en mayo 2019, https://doi. org/10.1177\%2F0042098019839886. Por su parte, la revista Espaces et Sociétés, en 2018, dedica el no 174 al tema financer la ville, les mutations des circuits de production du logement, en tanto que la misma revista dedica su n 170 de 2017 al tema logement et inégalités. 
decir, de lo vivido) (Seamon, 2007; Lindón, 2019), y no tanto desde las perspectivas de la movilidad espacial en sí misma ${ }^{3}$ : un mismo desplazamiento en cierto tiempo puede configurar una experiencia particular para cierta persona y una muy diferente para otra que viaja junto a la primera. En esas diferencias podrán mediar las rememoraciones que cada una realiza de otros desplazamientos vividos, en el mismo trayecto o en otros, aunque también podrían configurar la experiencia en cuestión otras dimensiones de ese presente vivido o de situaciones pasadas que, de alguna forma, la persona conecta con el desplazamiento actual.

Estas dos entradas analíticas llevan consigo una inversión del lugar de observación ${ }^{4}$ : antes que en-

3 Estudiar la movilidad espacial en sí misma es una forma de destacar la materialidad y sus flujos, considerando que lo material resulta de procesos históricos que conllevan formas sociales no solo actuales sino también pasadas, pero vigentes en el presente como formas espaciales, es decir, como tiempo pasado que deviene en espacio presente, en las rugosidades y las inercias dinámicas del espacio (Santos, 1990). En cambio, hacerlo en términos de la experiencia espacial supone preguntarse, por ejemplo, cómo viven distintos sujetos sociales un mismo desplazamiento, con la materialidad que lleva consigo la movilidad, pero también con lo no material que cada uno le agrega, de acuerdo con su memoria espacial de otras experiencias, sus ideas, creencias, fantasías y horizontes de sentido con los que enfrenta la cotidianidad (Ley \& Duncan, 1993). Las palabras de Guy Di Méo también enfatizan esta articulación entre lo material y lo vivido o la experiencia de lo material: "su doble naturaleza, objetiva y cognitiva, real y representada (también simbólica)" (1999, p. 75). En última instancia, estas palabras asumen el pensamiento de Godelier (1989), según el cual la realidad se constituye por lo ideal y lo material como dos aspectos indisociables de un todo llamado realidad.

4 La expresión "inversión" ha sido utilizada de manera pionera por la geógrafa francófona Renée Rochefort cuando, en los años sesenta del siglo XX, iniciaba la geografía social francófona. Esta autora planteaba la necesidad de invertir el orden de los factores entre el espacio y la sociedad, es decir, partir de la sociedad y no del espacio, como tradicionalmente hacía la geografía. Rochefort decía: "la geografía social comienza con la inversión del orden de los factores [entre el espacio y la sociedad], una inversión del interés" (Rochefort, 1963, p. 20). En estricto sentido, Renée Rochefort comienza a plantear la inversión de los factores con anterioridad, en los años cincuenta, en el contexto de su investigación dedicada a la actividad laboral como transformadora del territorio en el caso de Sicilia (Rochefort, 1961). focar la periferia desde la mirada aérea ${ }^{5}$ con un fuerte énfasis en la materialidad (las formas espaciales), se observa la periferia desde la perspectiva de los sujetos que la habitan. El punto de vista del habitante implica que los lugares se perciben desde la posición espacial del sujeto (De Castro, 1997) ${ }^{6}$ y lo percibido se tamiza por los "mundos interiores" del sujeto (Dardel, 1990), que usualmente son singulares ${ }^{7}$. Dicha inversión es una forma de revisitar la periferia de manera próxima, es decir, en escalas geográficamente grandes ${ }^{8}$.

Con este rumbo, se plantea una aproximación a la periferia vivida en clave de la experiencia espacial de habitarla. Por lo tanto, se asume una inversión espacial del punto de vista: se transita del espacio periférico a vuelo de pájaro (la morfología urbana) al espacio periférico vivido. Aunque esta inversión también conlleva otra de carácter temporal: antes que pensar el tiempo de la periferia como el de los procesos de expansión urbana, es decir, como procesos históricos, se lo considera como el tiempo vivido cotidianamente en la periferia9 . A ello se suma una tercera inversión de corte más metodológico, que es el análisis de la periferia a partir de las narrativas de los habitantes del lugar, antes que

5 Paul Claval (1999) ha identificado la mirada aérea con la metáfora icariana.

6 Esta posición, usualmente, es perpendicular a la superficie terrestre y no a vuelo de pájaro.

7 Lo singular refiere, en este texto, a especificaciones de lo social: ni lo único ni lo social.

8 Cabe subrayar que se utiliza la expresión "escalas grandes" en el sentido cartográfico: grande por el nivel de detalle (por la proximidad al fenómeno observado), y pequeño por el tamaño del territorio en cuestión, por el acercamiento mismo.

9 Esto se funda en la perspectiva dardeliana según la cual "Un mismo lugar es distinto para el nómada y para el sedentario" (Dardel, 1990, p. 46), de modo tal que se habla del espacio vivido en términos de la realidad geográfica externa a la persona, la que se puede percibir (el espacio percibido de Di Méo, 1991), pero reconfigurada por los mundos interiores de las personas (memoria, ideas, creencias, imaginación, etc.). 
el usual análisis de la periferia con base en datos agregados o bien en la observación del fenómeno periférico. En suma, el acercamiento planteado supone una múltiple inversión del punto de vista en beneficio de una microperspectiva de la periferia metropolitana, que en este caso no se limita a la mirada de tipo etnográfico en el sentido tradicional de esta expresión; más bien se desarrolla una microgeografía de la vida cotidiana.

Asimismo, cabe subrayar que este texto se limita a presentar un andamiaje teórico-metodológico para estudiar las periferias metropolitanas. Dicha perspectiva se ha ido configurando progresivamente en el contexto de diversos estudios empíricos realizados con anterioridad y referidos a la periferia oriental de la ciudad de México. En otras palabras, con cierta perspectiva epistemológica fundada en la inversión del punto de vista, el análisis de lo empírico generó la propuesta teórico-metodológica, que recién en esta ocasión es presentada como tal. El andamiaje teórico-metodológico es de tipo multidimensional y particularmente orientado a la comprensión de la dimensión espacial de las periferias metropolitanas recientes, que han surgido en antiguas tierras rurales que quedaron vacantes y luego fueron fraccionadas y ocupadas para uso habitacional bajo el signo de las carencias y la pauperización. Esto implica que este esquema quizás podría contener alguna pista pertinente para estudiar otros tipos de periferias, como aquellas que se configuran cuando la expansión urbana integra antiguas localidades o bien aquellas otras que emergen en tierras vacantes, pero que son manufacturadas para clases medias o medias altas que buscan alejarse de la vida central. Pero solo serían mínimos elementos los que pueden transferirse a otros tipos periféricos ya que no es un esquema de análisis desarrollado para esos otros tipos de peri- ferias metropolitanas, sino para aquellas que han sido producto de procesos de autoconstrucción y discursivamente se han configurado como territorios del sufrimiento, el aislamiento, las carencias y, a veces, de los logros lentos.

Para ello, en la primera parte se aborda la periferia como fragmentos densos que expresan estados inestables de lo urbano, siempre marcados por la tensión entre el cambio y el orden periféricos. Luego, la propuesta explora el abordaje de la periferia vivida a partir del análisis de las experiencias espaciales de los habitantes y sus biografías. Ello se revisa a través del anclaje periférico de los eventos biográficos de los habitantes de estos territorios, sean eventos individuales o familiares. Y por otro lado, se aborda la configuración de cronotopos periféricos en las narrativas locales como parte del proceso de construcción del sentido del lugar que se habita, en este caso, de la periferia habitada.

\section{La periferia como un estado inestable de la ciudad vivida}

La expresión "periferia de la ciudad" da cuenta de la relación de un territorio con otro dentro de un continuo urbano en expansión. Por ello, se trata de una voz esencialmente relacional: para que exista una periferia debe existir un centro. En otras palabras, un territorio solo puede ser periférico con relación a otro central. A pesar de esas bases semánticas del vocablo, la periferia de las ciudades se ha constituido crecientemente en una voz que refiere a un territorio en sí mismo. En este sentido, es posible explorar su carácter relacional internamente o de manera intraperiférica, es decir, con relación a las dinámicas internas. Y dentro del amplio espectro de dinámicas intraperiféricas, aquí se 
consideran las que fluyen entre lo que permanece y lo que cambia en estos territorios.

Las dinámicas entre lo que permanece y lo que cambia son indisociables del hecho de que las periferias son parte de la ciudad y por ello tienden a reproducir las tendencias homogeneizantes propias de lo urbano. Esto puede referir tanto a lo que permanece o a lo que se aspira que permanezca. Al mismo tiempo, las periferias son diferentes de la ciudad consolidada aun cuando sus particularidades se mantengan solamente durante cierto tiempo, es decir, sean transitorias. Esto otro da cuenta de lo que cambia.

La tensión entre el cambio y el orden periférico tiene tanto peso porque estos territorios surgen del cambio urbano que representa la expansión urbana, la ciudad viva que desborda sus límites y se extiende más allá de ellos transformando los territorios en los que se despliega: las periferias surgen como la prolongación de la ciudad que aún no lo es enteramente, pero ahora en otro territorio, es decir, donde faltan condiciones para ser ciudad, pero se espera que se las alcance y así llegue a ser ciudad propiamente dicha. Cuando lo que faltaba se alcanza, el territorio periférico deviene parte intrínseca de la ciudad misma y deja de ser periferia. En esos casos, este estadio transitorio de lo urbano que se denomina periferia vuelve a emerger en otro lugar más externo a la ciudad.

Este proceso urbano, tan conocido y extensamente abordado por los estudios urbanos latinoamericanos ${ }^{10}$, lleva consigo cambio y permanencia:

10 Solo como algunos ejemplos recientes de este tipo de abordaje se pueden recordar los trabajos de Rodríguez (2000), Bazant (2008), Vidal-Koppmann (2008) y Carrión (2012). una vez que esos territorios cambian por ser alcanzados por la expansión de la ciudad, en ellos se empieza a reproducir el orden urbano instaurado desde tiempo antes en la ciudad más central. Por ejemplo, la extensión de los servicios urbanos en un nuevo territorio periférico puede ser vista como la búsqueda del orden urbano instaurado previamente en la ciudad central que se busca reproducir en la nueva periferia, y se aspira a que ese orden permanezca. En otras palabras, es un cambio respecto a la situación periférica inicial, pero se orienta a reproducir el orden urbano conocido.

Esto es parte fundamental de la dinámica que anima a las periferias urbanas. Por ello, la periferia, además del contenido relacional, también lleva en su esencia el carácter transitorio o inestable que es propio del cambio y también se tensa entre esa fugacidad y su estabilización, es decir, el orden urbano que tiende a reproducirse, en buena medida, desde lo cotidiano.

Estos procesos de cambio territorial periférico no son continuos en el tiempo. En ellos se insertan pausas que detienen o demoran el cambio, aunque luego continúen las transformaciones. Dichas pausas pueden llegar a ser prolongadas en el tiempo o también breves. Además, estos procesos tampoco son homogéneos en su extensión: suele haber transformación en un lugar de cierta periferia y estancamiento en otro lugar colindante. Por ello se ha reiterado tanto la idea de la fragmentación de los territorios periféricos, y no solo dentro de la ciudad como un todo.

La tensión entre lo que permanece y lo que cambia en las periferias de las ciudades suele analizarse en la perspectiva de la consolidación urbana que 
expresa cambios en las formas espaciales y también en las relaciones sociales ${ }^{11}$. O bien, cuando esta tensión se inclina hacia las permanencias de las formas espaciales iniciales tiende a subrayarse la falta de progreso que demora la normalización del orden urbano. En ese tipo de aproximaciones, en términos espaciales se enfatizan las formas espaciales, en tanto que el tiempo se aborda como procesos históricos de las transformaciones materiales y en ocasiones es considerado como historia urbana, con su correspondiente correlato de cambios en las relaciones sociales y a veces también en las estructuras sociales.

Tanto el carácter relacional como el transitorio, que son propios de la periferia, se pueden explorar desde otros ángulos. Uno de ellos es a través de las experiencias espaciales de los sujetos que habitan la periferia, en las cuales emerge la tensión entre el cambio y lo que perdura. En este camino de lo vivido, que es el seguido en este texto, no sería factible estudiar toda la periferia de una ciudad, sino fragmentos que suponen sucesivos acercamientos en la escala de observación. También se puede considerar que estos fragmentos densos, en términos experienciales, deben ser identificados por los propios habitantes de la periferia. En otras palabras, no cabe una demarcación de los fragmentos inestables del territorio periférico realizada a priori por el estudioso del fenómeno. Más bien, estos fragmentos se van configurando en el habitar de las personas y son ellas quienes

11 Los cambios en las relaciones sociales de las periferias han sido extensamente documentados en los estudios urbanos latinoamericanos, por ejemplo, a través de la presencia de fenómeno conocido como clientelismo político, el caciquismo y los movimientos sociales que durante los años ochenta generaron extensos análisis de los movimientos urbanopopulares. Algunas referencias, entre muchas otras que no sería posible incluir aquí, son: Moctezuma (1984), Ramírez Saiz (1986), Massolo (1992), Ward (1977) y Cornelius (1973) los pueden demarcar en su propio discurso acerca de su vida en la periferia, o bien quien los estudia puede acercarse al fenómeno compartiendo la cotidianidad de estos sujetos.

\section{La periferia vivida}

La segunda entrada analítica para descifrar la periferia vivida son las experiencias espaciales con las que los sujetos van configurando el habitar periférico, es decir, la relación de las personas con lugares concretos de la periferia a lo largo de la vida. $\mathrm{Y}$ es en el cotidiano habitar que la periferia va siendo apropiada por sus habitantes, tanto materialmente como simbólicamente. De esta forma, la periferia es vivida en cada instante, pero todos esos fragmentos de tiempo están integrados en un todo, que es cada biografía. La referencia a lo biográfico aquí no se hace en el sentido de secuencias de eventos preidentificados de una vida linealmente organizada. La biografía se concibe como constelaciones infinitas de experiencias espaciales de los habitantes anónimos de la periferia que han sido guardadas en la memoria y pueden ser rememoradas y puestas en palabras. Dichas experiencias son las formas de vivir las situaciones que van configurando la vida cotidiana de las personas en la periferia, en las que se realizan ciertas prácticas con otros o ante otros, en determinados lugares y en fragmentos de tiempo específicos, bajo pautas o encuadres socioespaciales particulares.

La concepción de las experiencias espaciales de las personas como constelaciones de acontecimientos implica asumir que las vivencias en el lugar no son eventos aislados, sino que están unidos entre sí por una vida. El hilo conductor entre una y otra experiencia es la vida misma del sujeto. Analíticamente 
esto permite integrar la profundidad del tiempo vivido (los pasados y presentes) y también contiene claves acerca de la construcción social de los significados de la periferia como espacio de vivido: en ello radica la complejidad de la dimensión temporal de cada experiencia espacial periférica, ya que si bien siempre ocurre en un presente, en toda experiencia espacial se recrean y aplican saberes e imágenes incorporados previamente y también se pueden generar nuevos saberes y sentidos que podrán perdurar más allá de la experiencia misma para utilizarse en otras situaciones futuras, como ha enseñado la fenomenología social. Por ello, el presente de cada experiencia lleva consigo pasados y anticipa futuros. En esto se halla la densidad del presente vivido. Esta particularidad también configura el presente como un tiempo que se tensa entre el pasado y el futuro (Daniels, 1985).

Las experiencias pueden ser almacenadas por las personas, aun cuando sea parcialmente, bajo la forma de la memoria espacial de lo vivido. En estos términos, la biografía, que nunca será completa $^{12}$ y siempre será selectiva ${ }^{13}$, puede ser considerada como el nexo que une y entrelaza lo vivido en diversos lugares y diferentes momentos por cada persona. Paul Thompson (1988) ha destacado que los materiales biográficos pueden unir comunidades y generaciones. En este trabajo, la función de unir o entrelazar, propia de toda biografía, se considera particularmente con relación a las experiencias espaciales de cada biografía.

12 Nunca será completa porque se hace referencia a lo que se conoce como life story (y no life history), es decir, el relato de lo que la persona selecciona y pone en palabras (Bertaux, 2016).

13 El proceso de almacenar en la memoria lo vivido es selectivo, no se almacena todo, y el posterior proceso de rememorar y organizar discursivamente lo que se pone en palabras vuelve a agregar selectividad (Piña, 1989).
En otras palabras, la biografía de un sujeto une lugares que ha vivido y, además, una biografía se entrecruza con otras. Estas conexiones entre experiencias espaciales de una biografía y entre biografías constituyen tramas de sentido en términos generales y, de manera más específica, tramas de sentidos de los lugares o lo que también se ha denominado la "textura de los lugares" (Adams et al., 2001). De modo tal que la biografía narrada puede ser escudriñada para explorar las relaciones de las personas con los lugares que habitan y que han habitado.

Así, las biografías de los habitantes de la periferia llegan a constituir un trasfondo de infinitas experiencias y también de saberes elaborados y apropiados con los cuales los sujetos enfrentan, o enfrentaron, cada nueva experiencia espacial periférica. Así, las biografías van enhebrando las experiencias espaciales de los sujetos en la periferia y al mismo tiempo esas experiencias espaciales le van dando forma y fondo a cada vida, siempre territorializada. En esta perspectiva, la periferia se va conformando material y subjetivamente por el entrecruzamiento de múltiples biografías y experiencias espaciales.

Por todo lo anterior, se asume que la periferia no solo se configura como resultado de la consolidación material sino también de manera topológica, ya que cada nueva experiencia vivida va sellando el lugar en el que se ancló con la memoria de lo allí vivido, integrando al lugar los valores y sentidos puestos en juego en cada ocasión (Buttimer, 1976): este proceso es colectivo y multidimensional, a él contribuyen los diversos habitantes en función de sus propias biografías y así se van reconociendo/identificando con unos fragmentos de sentido, aunque no necesariamente con el todo, 
que indudablemente contiene territorios desconocidos y extranjerías para unos y no para otros.

Las biografías, como entrada analítica a lo socioespacial, no solo entrelazan lugares y vidas de diversos sujetos, sino que también se van anclando -o territorializando- en diversos lugares. David Seamon (1979) denominó arraigo a este proceso cuando tiene una fuerte componente corporal, y apropiación cuando está dominado por el sentido. Estas territorializaciones de las experiencias, con ambos matices (más corporales o más subjetivas), contribuyen a la construcción colectiva llamada periferia vivida. Por ocurrir todo ello en el constante devenir cotidiano, la periferia siempre se encuentra en condición inconclusa en términos existenciales y no solo en su materialidad. Así, la periferia vivida también es una periferia movimiento (Anderson \& Harrison, 2010) por los innumerables desplazamientos cotidianos, que siempre suponen la confrontación con diversas otredades (Stock, 2018), y también por la circulación de saberes espaciales, de discursos sobre los lugares y de afectividades entre los cuerpos periféricos ${ }^{14}$.

En este contexto, una forma de estudiar las tensiones entre lo que permanece y lo que cambia en la periferia es desde dos ejes analíticos. Al primero de ellos se lo denomina anclaje espacial de las biografías periféricas y al segundo se lo identifica como la fusión discursiva del tiempo y el espacio en cronotopos fundacionales de las periferias, que emergen en cronotopos de situaciones periféricas posteriores.

14 Esta concepción de periferia vivida como una periferia en movimiento adhiere a las teorías no representacionales (Thrift, 2008).

\subsection{El anclaje espacial de las biografías periféricas}

Todas las biografías se van inscribiendo en sus espacios de vida ${ }^{15}$ y esto es algo más complejo que la localización de los eventos de una vida en ciertas coordenadas. La inscripción de eventos biográficos en el territorio es un proceso por el cual el lugar le otorga características al evento biográfico y, al mismo tiempo, el acontecimiento - que es parte de una trayectoria de vida - marca y engrosa el lugar en cuanto a significados, memoria, valores. A ello se le denomina anclaje espacial. Dado que los eventos biográficos, en parte, se relacionan con las etapas de la vida, en el marcaje que hacen las vidas de los lugares (y lo inverso) media el tiempo biográfico. Así, algunos lugares se identifican con sucesos de la infancia de ciertas personas, de la juventud, de la vejez, o bien ciertos lugares resultan más afines para unas etapas de la vida y no para otras.

De esta forma, los anclajes de las biografías en la periferia constituyen una vía metodológica para comprender la integración del sujeto habitante con las otredades que suponen sus espacios de vida y su tiempo biográfico, con los lugares de la periferia y la temporalidad de los procesos periféricos. Asimismo, cabe considerar que el anclaje territorial de las biografías puede darse en los espacios domésticos (propios o no) de los habitantes de la periferia, en las calles o en espacios semipúblicos o híbridos. En otros términos, el anclaje no es exclusivo de los espacios más íntimos.

15 Se denomina espacio de vida al espacio percibido en la cotidianidad (es decir, reconocido sensorialmente por poseer materialidad) y al espacio practicado o espacio de las prácticas (Di Méo, 1991). 
Así, puede resultar pertinente metodológicamente explorar acontecimientos territorializados de una etapa biográfica de las personas a la luz de la consolidación urbana de la periferia en cuestión o de las carencias materiales de dicho territorio. Este tipo de periferias metropolitanas generalmente son habitadas por núcleos familiares, no obstante el anclaje de lo biográfico en el territorio puede ser individual, familiar o relativo a otros grupos sociales. Un ejemplo reiterado de los anclajes biográficos de tipo individual es el de las agresiones sufridas por mujeres en ciertos lugares del espacio público. Como ejemplo de anclajes familiares de lo biográfico se pueden mencionar los discursos en donde se señala, por ejemplo, que los hijos mayores de cierto núcleo familiar fueron a la única escuela del entorno periférico que existía en ese momento, en tanto que, con los hijos más pequeños, el mismo núcleo familiar pudo escoger la escuela porque ya existían varias. En otras ocasiones los discursos enlazan eventos festivos de la familia con las condiciones propias de la consolidación periférica: por ejemplo, ciertas fiestas familiares se tenían que hacer en las calles porque la casa familiar no permitía otra opción, en cambio otras fiestas familiares posteriores se pudieron hacer dentro de la casa cuando ya estaba avanzada la construcción. Estos anclajes periféricos de las biografías van enlazando la trayectoria de una familia o la biografía de un sujeto con el proceso de consolidación de la periferia misma. El entrelazamiento entre esos dos niveles -en principio, distantes-, en estricto sentido, ocurre cuando se construye la narrativa de vida.

Por lo anterior, una forma de constituir en objeto de estudio estos anclajes de las vidas en la periferia es a través de las narrativas de sus habitantes acerca de lo vivido en los lugares periféricos.
Así, la narrativa que el propio habitante del lugar reconstruya sobre momentos de su vida en la periferia se constituye en una mediación para conocer el anclaje espacial ${ }^{16}$. No obstante, el registro narrativo de lo vivido por cada sujeto en sus espacios de vida no es idéntico a los eventos vividos, más bien constituye una versión de aquella parte de lo vivido que el sujeto puede poner en palabras y organizar discursivamente de formas particulares en cierto presente (Piña, 1989; Vivero García, 2002). Si bien una lectura rápida de ello podría considerar que las inscripciones espaciales de lo vivido solo remiten a la indicación del lugar en donde ocurrió el evento (la localización del evento), una lectura más profunda suele mostrar la imbricación entre el lugar y el acontecimiento biográfico: en esa articulación suele ocurrir la transferencia del sentido que tuvo el acontecimiento vivido al lugar en el cual ocurrió y también puede emerger la influencia que el lugar tuvo en darle sentido al episodio biográfico que en él sucedió. Este proceso de unir narrativamente el evento y el lugar tiene la capacidad de profundizar el anclaje territorial ya que la experiencia es narrada como una vida que se detiene - se hace una pausa en el camino de la biografía - en cierto lugar para acontecer allí de cierta forma, tomar elementos del lugar para la vivencia en cuestión y dejar la vivencia como una huella del lugar. En otras palabras, la fusión del evento y su lugar se completa en la construcción narrativa y no en el momento en el que ocurrió el evento. De esta forma, para la configuración de la periferia como territorio vivido (o periferia vivida) no es suficien-

16 Existen otras formas de acceso a lo vivido, por ejemplo, las fotografías familiares acompañadas de relatos sobre las mismas pueden ser otra puerta de acceso para los estudiosos de la periferia en esta perspectiva (Mannay, 2017). En esos casos, la incorporación del relato acerca de la fotografía es fundamental porque es en él donde se produce la rememoración y la nueva interpretación de lo vivido. 
te con identificar el evento, se requiere la posterior narrativa sobre el mismo porque es allí cuando se funde el lugar y el sentido.

Por otra parte, el anclaje espacial de las biografías no solo se configura por lo que acontece y su lugar, sino también por los saberes espaciales de sentido común que los sujetos movilizan en el acontecimiento allí vivido. Estos saberes son de dos tipos: aquellos que el sujeto poseía con antelación a la experiencia espacial en cuestión y los que produce en esa nueva circunstancia de su vida. Los nuevos saberes espaciales siempre se producen a partir de otros que han sido configurados con antelación, pero que son puestos en movimiento en el presente. La integración de los saberes espaciales en el proceso de anclar los acontecimientos vividos en cierto lugar también pone en evidencia que el lugar no es el simple locus en el cual se localiza cierto acontecimiento. Los saberes espaciales muestran que la inscripción de lo vivido en un lugar particular viene asociada al saber actuar en el lugar, saber qué hacer o qué no hacer en ese lugar durante dicha experiencia o cómo hacer lo que la situación amerita. Así, tenemos que los acontecimientos biográficos que han quedado asociados con ciertos lugares también llevan consigo la identificación de ciertas prácticas desarrolladas en el lugar, que en última instancia son indisociables de saberes o conocimientos de sentido común.

En el caso de las periferias, por su condición híbrida (entre la ciudad que aún no lo es enteramente y la ciudad que llega a tierras vacantes) e inestable (por la tensión entre el cambio y la permanencia), es frecuente que los habitantes posean saberes que no se aplican en la periferia o que resulten insuficientes o inapropiados para el nuevo espacio de vida periférico. Esto puede deberse a que estos su- jetos anteriormente habitaban áreas centrales de la ciudad o bien procedían de zonas rurales. En uno y otro caso, se experimenta una falta de armonía entre los saberes prácticos que se poseen y los que se requieren, que le genera al habitante cierta tensión con el lugar por esta peculiar disonancia cognitiva. Circunstancias de ese tipo pueden interpretarse como una suerte de experiencia de la "extranjería" en el sentido de Bégout (2005, pp. 26-27): esto es, la tensión que surge ante la nueva cotidianidad periférica, por ejemplo, sin los servicios básicos, cuando los habitantes del lugar solo disponen de saberes espaciales para resolver la vida práctica con dichos servicios y no en ausencia de ellos.

Si bien estos anclajes de lo vivido en lugares muy específicos de la periferia son individuales, en numerosas ocasiones trascienden ese nivel y se hacen parte no solo de la familia, sino también de la memoria colectiva del lugar (Javeau, 2000), sobre todo en el caso de experiencias espaciales de los inicios de la periferización, o bien en situaciones posteriores, pero que se tornan emblemáticas del habitar periférico ${ }^{17}$. Ello ocurre por varias circunstancias: por un lado, porque la experiencia anclada en el lugar puede haber sido vivida por varios sujetos. Y, por otro lado, porque ciertas experiencias

17 De acuerdo con Heidegger, el habitar expresa el permanecer en los lugares en condición de libertad, protección, con satisfacción y sin amenaza. En esta perspectiva, el habitar es un vínculo armónico entre el hombre y el medio, y refiere a la existencia espacial del ser humano Heidegger (1975 [1954]). Sin embargo, en las perspectivas más contemporáneas, el habitar se ha deslizado hacia los modos de vida y, de manera más amplia, hacia a la vida cotidiana en las ciudades. En este sentido, el habitar un lugar supone la realización de ciertas prácticas y también lleva consigo saberes acerca del lugar y lo que allí se hace o cómo se hace (Stock, 2004; Giglia, 2012; Lussault \& Stock, 2010; Radkowski, 2002; Lazzarotti, 2006). Así, la relación de las personas con los lugares (el habitar) está mediada por las prácticas y los saberes del lugar y de las prácticas locales. Estas mediaciones también pueden ser de orden material, como los objetos de los que disponen los sujetos o los que están en el lugar. 
ancladas en lugares particulares de la periferia son narrativizadas ${ }^{18}$, y así circulan como discursos sociales y muy diversos sujetos asumen el anclaje de la experiencia en cuestión con el lugar, aun sin haber conocido al sujeto o los sujetos que la protagonizaron. Asimismo, la narrativización contribuye a otro proceso: la simbolización de ciertos lugares de la periferia como expresión materializada de algunos acontecimientos que pueden ser parte de una biografía y también de otras. En este tipo de periferias, en buena medida estas narrativizaciones se vinculan a eventos trágicos, indisociables de las carencias materiales del lugar, y así contribuyen a configurar la periferia por la activación de los mecanismos del lenguaje, como la reflexividad que lleva que al decir algo, se hace ${ }^{19}$.

En este tipo de procesos narrativos se pone en movimiento la configuración de la identidad del lugar $\mathrm{y}$, al mismo tiempo, la identificación de sus habitantes con el lugar (Duncan \& Duncan, 2001). Este es un proceso doble: el evento narrativizado (no necesariamente trágico) le da una particularidad al lugar, y, al mismo tiempo, las personas desarrollan una relación de espejo con el lugar.

En las narrativas de experiencias periféricas fuertes también suele presentarse cierto tono épico o de

18 La narrativización "es un recurso literario que consiste en omitir la atribución de lo dicho a una voz o un actor en particular. Al omitirse que algo de lo que se está tratando ha sido dicho por alguien en particular, el efecto que se alcanza es que lo dicho aparece como 'algo dado' más allá de una voz o de otra. Esta estrategia discursiva borra u omite un aspecto importante, como es el reconocimiento que lo dicho no es algo dado, sino algo que fue dicho de esa forma por alguien y en ciertas circunstancias. Cuando en un discurso se hace referencia a un actor al que se le atribuye lo dicho, en cierta forma lo dicho pierde fuerza porque se ingresa al terreno del nivel de credibilidad que tenga el actor que lo ha dicho, o bien se entra en el campo de que, a lo dicho por alguien, se puede oponer lo dicho por otra voz" (Lindón, 2007, p. 128).

19 Se hace referencia a la reflexividad en el sentido etnometodológico (Garfinkel, 2006). epopeya, en donde se dibuja un héroe y la historia que se cuenta involucra a otros, a una comunidad, así como valores compartidos en ese mundo social. Esto resulta particularmente frecuente en el caso de los pioneros o primeros pobladores periféricos, precisamente por haber enfrentado las adversidades propias de las carencias iniciales de un territorio periférico.

Si bien las periferias suelen ser dinamizadas por procesos de transformación material que en ciertos momentos se aceleran, los anclajes de lo vivido llegan a perdurar aun cuando materialmente los lugares sean renovados profundamente e incluso permanecen en las narrativas locales cuando se han perdido totalmente las huellas materiales del lugar que fue o del evento allí ocurrido. De esta forma, los anclajes en lugares particulares de los acontecimientos que integran las vidas periféricas interactúan con la tensión entre lo que permanece y lo que cambia en las periferias. Y en esa interacción resultan claves las dimensiones biográficas y discursivas.

\subsection{Los cronotopos periféricos}

Las periferias metropolitanas de autoconstrucción no tienen una fecha precisa en la que hayan iniciado, ni siquiera cuando haya habido algún evento desencadenador como puede ser una invasión o usurpación de terrenos. No es posible que tengan una fecha precisa que marque su inicio porque las periferias se construyen en el cotidiano devenir de llegar a habitar un territorio antes deshabitado. A pesar de esa ambigüedad temporal que lleva consigo lo cotidiano en estas periferias, es frecuente que los pioneros de estos territorios metropolitanos vayan configurando culturalmente un tiempo fundacional, de modo tal que el modelado cultural 
de los tiempos iniciales llega a constituirse en una necesidad que es vivida como algo tan relevante como los servicios y los equipamientos urbanos. Posiblemente, dicha necesidad cultural se relaciona con la búsqueda del reconocimiento social de una nueva existencia en el tejido urbano. En cuanto a la dimensión fáctica, los tiempos iniciales de las periferias suelen identificarse con ciertos eventos, como pueden ser los primeros fraccionamientos o lotificaciones de tierras que antes eran rurales o la construcción de las primeras viviendas. Sin embargo, esos eventos suelen ser cronológicamente difusos y ello atenta contra la identificación precisa de los inicios de la periferia en cuestión. En cambio, los tiempos fundacionales suelen empezar a configurarse más nítidamente como un campo de enunciados ${ }^{20}$ que repiten unos y otros, es decir, los habitantes locales.

Dicho campo semántico de enunciados se identifica por la circulación de ciertos conceptos de sentido común que se van entrelazando con el cotidiano quehacer en el nuevo territorio. Por ejemplo, en la periferia oriental de la ciudad de México de los años ochenta del siglo XX, algunos de esos conceptos que fueron integrando y demarcando el campo semántico de enunciados que permitiría luego configurar los tiempos fundacionales fueron las expresiones lodazal, zanjas, charcos, remolinos de viento y tierra, caminatas extensas, sufrimiento diario y falta de agua potable junto con el exceso de agua de inundación. Asimismo, estos campos de enunciados se van consolidando y complejizando con la incorporación de hitos topológicos que singularizan el campo de enunciados propio de la periferia en cuestión. Los hitos topológicos refie-

20 Se retoma la expresión campo de enunciados en el sentido foucaultiano (Foucault, 1968).

Vol. $25 \mathrm{~N}^{\circ} 2$

julio - diciembre de 2020

pp. 15-33 ren a lugares fuertes para la vida cotidiana local, reconocidos como tales por diversos habitantes de la periferia en estudio y casi siempre se asocian a dificultades, obstáculos e incluso tragedias. Si bien cualquier campo semántico de enunciados de lo urbano tiene hitos topológicos de diversa naturaleza (por ejemplo, lugares de diversión, lugares de cierto estilo, lugares de olores peculiares, colores o sonidos, entre otros), los que son propios del tipo de periferia referida y de sus inicios casi siempre dan cuenta de topologías del sufrimiento y las carencias.

En este horizonte, dichos campos semánticos de enunciados y sus hitos topológicos llegan a configurar narrativamente un tiempo fundacional de la periferia que termina siendo más robusto que la ausente referencia cronológica al inicio. Esta construcción discursiva de lo fundacional no se limita a la dimensión temporal de la vida social local, sino que también se integra en ella la dimensión espacial. Por ello, puede resultar iluminador explorar los tiempos fundacionales de las periferias bajo la metáfora del cronotopo bajtiniano. Para ello, se puede recordar que, de acuerdo a Bajtín, "En el cronotopo [artístico literario] tiene lugar la unión de los elementos espaciales y temporales en un todo inteligible y concreto. El tiempo se condensa aquí, se comprime [...]; y el espacio, a su vez, se intensifica, penetra en el movimiento del tiempo [...], de la historia. Los elementos del tiempo se revelan en el espacio, y el espacio es entendido y medido a través del tiempo" (Bajtín, 1989, p. 237). En esencia, el cronotopo es la conexión narrativa del tiempo y el espacio que organiza el mundo de la vida cotidiana (las prácticas) de algún héroe anónimo. 
En la periferia oriental de la Ciudad de México de los años ochenta del siglo XX, los estudios urbanos de carácter empírico han puesto en evidencia elementos que permiten identificar el cronotopo fundacional en torno a un espacio extenso, sin límites, vacío y sin fronteras al cual se enfrentaba el habitante-héroe y lo combatía. Estos atributos de la espacialidad se vivían de forma negativa: el sujeto en ese espacio no se podía orientar espacialmente porque todo era homogéneo y vacío. La homogeneidad derivaba de la ausencia de construcciones, entonces no solo impedía la orientación, que le resta certezas espaciales básicas a toda persona, también constituía la ausencia de todo lo necesario para la cotidianidad. La extensión también representaba el cotidiano sacrificio para desplazarse y llegar a la ciudad misma para resolver la vida práctica. Al mismo tiempo, la amplitud espacial también era vivida como la posibilidad de sufrir agresiones y no poder contar con la asistencia de otros por las distancias vacías y enormes que separaban a una persona de otra. En este cronotopo, los eventos cotidianos son inevitablemente fugaces (ocurren en cierto momento, que se desvanece rápidamente). Sin embargo, la espacialidad les otorga profundidad y sobre todo les da permanencia, como si el tiempo no pasara y quedara detenido en el lugar del acontecimiento. Por ejemplo, la temporalidad fugaz de un acto de violencia sufrido en la calle se puede condensar en un instante, mientras que su espacialidad se intensifica en la simbolización duradera de la calle (o un fragmento de esta) como un lugar de violencia y también como el lugar en el cual las prácticas cotidianas de desplazamiento integran rutinas protectoras para convivir con la violencia. También es posible que el acto de violencia circule entre varias personas presentes, de cuerpo en cuerpo, aun cuando solo una de ellas sea agredida. El héroe —o, más fre- cuentemente, la heroína - de la historia es quien sortea esa espacialidad amenazante con estrategias y tácticas defensivas, aunque ese papel de heroína también se relaciona con la narrativa construida a posteriori acerca de la experiencia que circuló y afectó a varias personas. En este tipo de situaciones no emerge el cronotopo fundacional, sino una derivación o reconfiguración de este en un tiempo posterior al fundacional, pero que extiende los tiempos fundacionales. Así, en el cronotopo derivado, el espacio fuerte de la periferia vacía de los inicios (por falta de servicios, infraestructuras, construcciones) se reconfigura como un espacio también fuerte, pero que se ha ido llenando de acontecimientos y sobre todo de experiencias de sufrimiento y violencia. El espacio sigue siendo lo que concreta la permanencia, siempre a través de la mediación de la discursividad.

La integración de la metáfora bajtiniana del cronotopo como herramienta analítica de los tiempos fundacionales de este tipo de periferias permite explorar la fusión del espacio, el tiempo y el héroe $^{21}$ : solo es posible postular un cronotopo cuando el tiempo — en este caso, el fundacional- se comprime y se imprime en el territorio periférico, mientras tanto el espacio periférico suele intensificarse, casi siempre con su desolación, vacío, carencias y ausencias. Así, el cronotopo -y en particular el de carácter fundacional o sus derivados - suele evidenciar el modelado que ha hecho y sigue haciendo el espacio del tiempo, y por ello resulta clave en la configuración identitaria de los habitantes. También es posible retomar esta metáfora bajtiniana para otros cronotopos periféricos y no solo los fundacionales. Frecuentemente el

21 El héroe (de la novela), en el sentido bajtiniano, refiere a una perspectiva sobre el mundo, sobre sí mismo y sobre la situación vivida (Bajtín, 1999). 
cronotopo fundacional se reproduce y emerge en otros cronotopos más específicos, más pequeños y de tiempos posteriores al fundacional, pero con la misma esencia. Esta capacidad activa del lenguaje práctico de unir el tiempo y el espacio, pero también el decir y las prácticas, permite que, aun cuando los cambios y las transformaciones materiales de la periferia analizada sean acelerados, las narrativas y los cronotopos que tejen tiendan a fijar los eventos en el espacio y darles permanencias de largo plazo.

De esa forma, la figura del cronotopo - sea fundacional o también su recreación en espacios/tiempos fuertes posteriores a los tiempos fundacionales - deviene en una herramienta de análisis de la periferia vivida que lleva consigo varias inversiones del punto de vista del estudioso. La posibilidad de revisitar la periferia desde esta pauta al menos implica invertir la perspectiva aérea por otra de tipo experiencial. También supone la inversión del punto de vista de la periferia como materialidad al de la periferia como materialidad vivida. De igual forma, supone invertir la estrategia metodológica de medición de las formas espaciales hacia la interpretación de narrativas de vida espaciales, siempre en proceso de hacerse.

En otras palabras, los análisis más usuales de las periferias destacan la centralidad del tiempo sobre el espacio y esto no es ajeno al hecho de que las periferias suelen estudiarse como procesos de expansión urbana. Así, mientras que la figura del cronotopo es una clara inversión de la perspectiva de estudio porque antes que pensar la periferia desde los procesos de expansión de la ciudad con énfasis en el tiempo se destaca la centralidad del espacio en la periferia, el cronotopo narrativamente construido muestra la subordinación del tiempo al espacio.

Vol. 25 N. ${ }^{\circ} 2$

julio - diciembre de 2020

pp. 15-33

\section{Reflexiones finales}

La forma presentada de aproximación a la periferia solo muestra un camino que evita la reificación de este territorio, tan frecuente en aquellos estudios urbanos que priorizan las formas espaciales. Tampoco se omiten las formas espaciales, solo se gira la mirada desde la materialidad insoslayable de la periferia a las diversas formas de vivirla (Lancione \& McFarlane, 2016). Aun con estas aclaraciones, cabe subrayar que esta propuesta no aspira a ser considerada como una serie de pasos a replicar en diversos casos empíricos.

De igual forma, se subraya que las dimensiones analíticas aquí consideradas — necesariamente inconclusas - no se agotan en sí mismas ni son las únicas pertinentes. Cada una de estas dimensiones se puede ir abriendo en categorías más próximas al fenómeno estudiado. Aquí se ha intentado mostrar el potencial analítico de integrar en los estudios sobre la periferia cuestiones como las experiencias espaciales, las narrativas de los habitantes del lugar y los anclajes de las biografías individuales y familiares en la periferia, así como los cronotopos configurados en el discurso cotidiano de quienes habitan estos lugares. Sobre todo, interesan las relaciones tejidas entre todas estas dimensiones ya que la complejidad de las periferias amerita perspectivas relacionales y sensibles al movimiento en el sentido amplio - lo que integra el aspecto vitalista de la expresión - y no solo el movimiento como desplazamiento de un lugar a otro. Y tanto el devenir como el flujo constante de la vida periférica se pueden relevar mejor desde el mundo de la vida cotidiana.

La posibilidad de iluminar casos empíricos con estas dimensiones necesariamente requiere de la incorporación de otras categorías, casi siempre 
vinculadas a la vida cotidiana porque es en esta donde se configuran y movilizan las mencionadas cuestiones. Un ejemplo de las categorías que aquí no han sido abordadas a fondo, pero cuya integración es imprescindible en la puesta en movimiento de esta propuesta, son las prácticas espaciales.

En última instancia, la propuesta presentada esboza un acercamiento al urbanismo cotidiano periférico (los modos de vivir la periferia) a través de fragmentos densos de periferia (McFarlane, 2018). El giro hacia este tipo de urbanismo puede interpretarse como una reconfiguración cotidiana del orden urbano en fragmentos de espacio-tiempo que a veces transgreden, otras resisten y casi siempre resignifican la aparente homogeneidad aspiracional representada por el orden urbano consolidado. Esto genera fragmentos espaciotemporales inesperados, también inestables y fugaces, que pueden resultar invisibles para una observación poco profunda. Estos fragmentos inesperados se debaten entre el orden urbano y el cambio. Aunque en oca- siones el cambio solo busca alcanzar el orden (por ejemplo, con la extensión de los servicios urbanos o la reproducción de rutinas cotidianas), en otras es creador de nuevas formas de vida, de nuevos urbanismos cotidianos. En última instancia, el reconocimiento y el análisis de fragmentos densos muestra el potencial analítico de las perspectivas topológicas (McFarlane, 2016) que exploran los procesos de inscripción de los eventos biográficos en los lugares de la periferia y la acumulación de significados, valores y memoria en los lugares cotidianos, así como el modelado discursivo de esta densidad. No ha sido azaroso que lo topológico haya resultado tan soslayado por largo tiempo en los estudios urbanos y en particular en los estudios de la periferia: ese olvido reiterado no parece ajeno a la centralidad que tuvieron las aproximaciones corológicas, más interesadas en la extensión del continuo urbano y a veces en su fragmentación (McFarlane, 2018) que en los fragmentos densos en sí mismos. 


\section{Referencias}

Adams, P. C., Hoelscher, S. \& Till, K. E. (Eds.) (2001). Textures of Place: Exploring Humanist Geographies. University of Minnesota Press.

Anderson, B. \& Harrison, P. (2010). Taking-Place: Non-Representational Theories and Geography. Routledge.

Bajtín, M. (1989). Teoría y estética de la novela. Taurus.

Bajtín, M. (1999). Estética de la creación verbal. Siglo XXI.

Bazant, J. (2008). Procesos de expansión y consolidación urbana de bajos ingresos en las periferias. Bitácora, 2(13), 117-132.

Bégout, B. (2005). La découverte du quotidien. Allia.

Bertaux, D. (2016). Récit de vie. Armand Colin.

Buttimer, A. (1976). Grasping the dynamism of lifeworld. Annals of American Association of Geographers, 66(2), 277-292.

Carrión, F. (2012). La forma urbana de Quito: una historia de centros y periferias. Bulletin de l'Institut Français d'Études Andines, 41(3), 503-522.

Claval, P. (1999). La geografía cultural. Eudeba.

Cornelius, W. (1973). Contemporary Mexico: A Structural Analysis of Urban Caciquismo. En R. Kern (ed.), The Caciques: Oligarchical Politics and the System of Caciquismo in the Luso-Hispanic World. University of New Mexico Press.

Daniels, S. (1985). Arguments for a Humanistic Geography. En R. J. Johnston (ed.), The future of Geography, pp. 143-158. Methuen.

Dardel, E. (1990). L'homme et la terre, Nature de la réalité géographique [1a ed. de 1952]. Editions du CTHS.

De Castro, C. (1997). La geografía en la vida cotidiana. Ediciones del Serbal.

Di Méo, G. (1999). Géographies tranquilles du quotidien. Une analyse de la contribution des sciences sociales et de la géographie à l'étude des pratiques spatiales. Cahiers de géographie du Québec, 43(118), 75-93. https://doi.org/10.7202/022788ar.

Di Méo, G. (1991). L'Homme, la société, l'espace. Anthropos.

Duncan, J. S. \& Duncan, N. G. (2001). Sense of place as a positional good: Locating Bedford in Space and Time. En P. C. Adams, S. Hoelscher \& K. E. Till (eds.), Textures of Place: Exploring Humanist Geographies (pp. 41-54). University of Minnesota Press.

Foucault, M. (1968). Las palabras y las cosas: una arqueología de las ciencias humanas. Siglo $\mathrm{XXI}$.

Garfinkel, H. (2006). Estudios en etnometodología. Anthropos.

Giglia, A. (2012). El habitar y la cultura: perspectivas teóricas y de investigación. CDMX: Anthropos, Universidad Autónoma Metropolitana Iztapalapa. 
Godelier, M. (1989). Lo ideal y lo material. Pensamiento, economías, sociedades. Taurus.

Heidegger, M. (1975). Construir, habitar, pensar. Revista Teoría, 5-6, 150-162. [Versión inicial de 1954, "Vorträge und Aufsätze", Verlag Günther Neske, Pfullingen y conferencia de 1951].

Javeau, C. (2000). Lugares de memoria individuales y estructuración de las interacciones acerca de los síndromes de Lamartine y de Proust. En A. Lindón (coord.), La vida cotidiana y su espacio-temporalidad (pp. 171-186). Anthropos.

Lancione, M. \& McFarlane, C. (2016). Life at the urban margins: Sanitation infra-making and the potential of experimental comparison. Environment and Planning A: Economy and Space, 48(12), 2402-2421. https://doi.org/10.1177/0308518X16659772.

Lazzarotti, O. (2006). Habiter: La condition géographique. Belin.

Ley, D. \& Duncan, J. (1993). Introduction. En J. Duncan \& D. Ley (eds.), Place/Culture/ Representation. Routledge.

Lindón, A. (2007). Colonización de la subjetividad espacial por el imaginario suburbano en las periferias de la ciudad de México. L'Ordinaire des Amériques, 207, 117-139.

Lindón, A. (2019). The lived city: everyday experiences, urban scenarios and topological networks, Geographica Helvetica, 74, 31-39. https://doi.org/10.5194/gh-74-312019.

Lussault, M. \& Stock, M. (2010). "Doing with space": towards a pragmatics of space. Social Geography, 5(1), 11-19. https://doi.org/10.5194/sg-5-11-2010.

Mannay, D. (2017). Métodos visuales, narrativos y creativos en investigación cualitativa. Ediciones Narcea, Ministerio de Educación Cultura y Deporte.

Massolo, A. (1992). Por amor y coraje. Mujeres en movimientos urbanos de la ciudad de México. PIEM, El Colegio de México.

McFarlane, C. (2018). Fragment Urbanism: Politics on the Margins of the City. Environment and Planning D: Society and Space, 36, 1007-1025. https://doi. org/10.1177/0263775818777496.

McFarlane, C. (2016). The Geographies of Urban Density: Topology, Politics and the City. Progress in Human Geography, 40, 629-648. https://doi. org/10.1177/0309132515608694.

Moctezuma, P. (1984). El movimiento urbano popular mexicano. Nueva Antropología, $6(24), 62-87$.

Piña, C. (1989). Sobre la naturaleza del discurso autobiográfico. Argumentos, 7, 131-160.

Radkowski, G. H. (2002). Anthropologie de l'habiter: Vers le nomadisme. PUF.

Ramírez Saiz, J. M. (1986). El movimiento urbano popular. Siglo XXI.

Rochefort, R. (1963). Géographie sociale et sciences humaines. Bulletin de l'Association des Géographes Français, 314-315, 18-32.

Rochefort, R. (1961). Le travail en Sicile : Étude de géographie sociale. PUF. 
Rodríguez, M. (2000). Periferia y suelo urbano en la Zona Metropolitana de la Ciudad de México. Sociológica, 15(42), 59-90.

Santos, M. (1990). Por una geografía nueva. Espasa Calpe.

Seamon, D. (1979). A Geography of the Lifeworld. St. Martin's Press.

Seamon, D. (2007). Interconnections, Relationships, and Environmental Wholes: A Phenomenological Ecology of Natural and Built Worlds. En D. Abram \& M. Geib (eds.), Phenomenology and Ecology, pp. 53-86. Duquesne University Press.

Stock, M. (2018). Habiter la ville en mouvements. En W. Asholt, E. Heurgon, P. OsterStierle \& M. Calle-Gruber (eds.), L'Europe en mouvement, vol. 1: À la croisée des cultures, pp. 145-154. Hermann.

Stock, M. (2004). L'habiter comme pratique des lieux géographiques. EspacesTemps. net. https://www.espacestemps.net/articles/habiter-comme-pratique-des-lieuxgeographiques/.

Thompson, P. (1988). La voz del pasado: historia oral, Valencia: Edicions Alfons El Magnànim.

Thrift, N. (2008). Non-representational theory: space, politics, affect. Routledge.

Vidal-Koppmann, S. (2008), La expansión de la periferia metropolitana de Buenos Aires. "Villas miseria" y "countries": de la ghettización a la integración de actores en el desarrollo local urbano. Scripta Nova: Revista Electrónica de Geografía y Ciencias Sociales, 245(42). http://www.ub.es/geocrit/sn/sn-24542.htm.

Vivero García, M. D. (2002). El discurso autobiográfico: ideología de la transparencia y mito de la autenticidad. Thélème, Revista Complutense de Estudios Franceses, 17, 283293.

Ward, P. (1977). Una comparación entre colonias paracaidistas y ciudades perdidas de la Ciudad de México. Hacia una nueva política. Investigaciones Geográficas, 8, 101-121. 